\title{
EDITORIAL
}

\section{How should we treat portopulmonary hypertension?}

\author{
M.J. Krowka and K.L. Swanson
}

$\mathbf{T}$ he development of pulmonary arterial hypertension in the setting of advanced liver disease (portopulmonary hypertension (POPH)) has certainly captured the attention of all liver transplant centres, but why? For patients with moderate-to-severe POPH (mean pulmonary artery pressure $(\mathrm{mPAP})>35 \mathrm{mmHg}$ and pulmonary vascular resistance (PVR) $>400$ dyne $\cdot \mathrm{s} \cdot \mathrm{cm}^{-5}$ ), the risk of intra-operative death and posttransplant hospitalisation mortality in otherwise acceptable liver transplant candidates is high [1]. The American Association for the Study of Liver Disease Practice Guidelines have espoused Doppler echocardiography screening for all liver transplant candidates [2]. However, once POPH is confirmed by right-heart catheterisation, what therapeutic approach should be considered? More generally, what should the approach be to treating $\mathrm{POPH}$, irrespective of liver transplant candidacy?

POPH is not simply a liver transplant issue, but that area of clinical medicine has raised awareness of this entity over the past few years. Recent evidence from France underscores the importance of POPH: it was the third-most common type of pulmonary hypertension seen in a consortium of 17 French hospitals [3]. The French evidence mirrors that of the Mayo Clinic (Rochester, MN, USA) over the past $10 \mathrm{yrs}$. POPH is the third-most common disorder documented in the Mayo Pulmonary Hypertension Clinic since 1996.

In the current issue of the European Respiratory Journal, REICHENBERGER et al. [4] describe the results of an uncontrolled study of 12 unstable POPH patients who received sildenafil ( $\leqslant 50 \mathrm{mg}$ three times daily) as either initial monotherapy $(n=6)$ or as an addition to existing prostacyclin therapy $(n=6)$. These patients had moderate-to-severe $\mathrm{POPH}$ as defined by mPAP and PVR. An additional 20 POPH patients who were stable or had mild POPH were excluded from the study (including three patients who were being treated with bosentan), so some degree of selection bias did exist. Sildenafil appeared to provide therapeutic benefit (decreasing $\mathrm{MPAP}$ and PVR) when evaluated at 3 months, but the haemodynamic benefit was not sustained at 12 months in seven patients, as measured by PVR, mPAP and cardiac index. Curiously, the 6-min walk distance did continue to improve at both 3 months and 12 months. This further illustrates the importance of longer-term follow-up in

Mayo Clinic, Rochester, MN, USA.

SUPPORT STATEMENT: Neither author has any financial arrangements that would affect this editorial. Specifically, they have no relationship of any type with the pharmaceutical company that produces and markets sildenafil.

CORRESPONDENCE: M.J. Krowka, Division of Pulmonary and Critical Care Medicine, Mayo Clinic E-18, Rochester, Minnesota 55905, USA. Fax: 1 5072664372. E-mail: krowka@mayo.edu pulmonary hypertension trials than the usual 12- to 16-week end-points. Compared with baseline, the small group who received combination therapy seemed to have the best benefit in terms of PVR at 12 months. Obviously, these trends are based on only a few patients (and subjective interpretation of the data), but the individual changes over time are quite heterogeneous and not necessarily apparent from looking simply at the group mean data.

Patients with POPH face several dilemmas, but two are worthy of comment in the context of the study by REICHENBERGER et al. [4]. First, POPH patients have not been included in randomised, controlled clinical trials that focus on pulmonary artery hypertension. Indeed, that was the case in the recent multicentre sildenafil SUPER trial [5], which involved 278 patients with pulmonary hypertension classified as idiopathic, associated with connective disease or occurring after surgical repair of congential systemic-to-pulmonary shunts. We have seen only two small (uncontrolled) trials using either i.v. epoprostenol or bosentan, respectively, to treat POPH patients. Both trials demonstrated pulmonary haemodynamic improvement up to $1 \mathrm{yr}$ following treatment $[6,7]$.

Secondly, what is "success" in treating POPH? Are 6-min walk data representative of therapeutic effect in patients with varying degrees of encephalopathy, significant ascites, anaemia and the pre-existing fatigue associated with liver disease? In the study by REICHENBERGER et al. [4], improvement was noted in nine out of 12 patients regardless of whether they received monotherapy or combination therapy. No Child class C patients were studied. No significant toxicity (worsening hepatic function or increased evidence of bleeding over 12 months) was reported at the sildenafil doses given, which is encouraging.

Is a further increase in cardiac output desirable when a hyperdynamic circulatory state already exists in advanced liver disease? Should we expect a decrease in both the MPAP and PVR? Is an isolated reduction in PVR acceptable? The data presented by REICHENBERG et al. [4] show various haemodynamic combinations of possible "response" to sildenafil. Not all observations were favourable, with decrease in cardiac index associated with increasing PVR occurring in three patients receiving sildenafil monotherapy. However, a decrease in both PVR and MPAP would be an optimal result in the current authors' opinion, and three patients demonstrated such change while on combination therapy. Finally, a decrease in PVR, but no change in mPAP (due to increased CO?), were noted $(n=3)$, associated with an improved 6-min walk and that would seem to be a favorable, albeit less optimal outcome. Perhaps success will ultimately be measured by 
right-heart function, survival and quality of life (or ability to safely conduct successful liver transplantation).

Finally, a thought about the sildenafil mechanism of action (enhancing nitric oxide-mediated vasodilatation) in the setting of a high-flow state caused by a vasodilated splanchnic circulatory state. If increased nitric oxide is the main splanchnic mediator, will increasing doses of sildenafil further impair systemic haemodynamics in patients with POPH? Further observations will be telling and additional sildenafil mechanisms of action may well complement the role of vasodilation.

In summary, portopulmonary hypertension is a vaso-obstructive process whose pathophysiology (in genetically susceptible patients) most likely involves a progression from a high-flow state to endothelial/smooth muscle proliferation, in situ thrombosis and plexogenic arteriopathy within the pulmonary arterial circulation [1]. Hence portopulmonary hypertension is more than simply vasoconstriction. The data of REICHENBERGER et al. [4], showing initial improvement at 3 months (yet worsening at 12 months) suggest that the vasodilation approach provided by sildenafil alone may not be the optimal treatment in moderate-to-severe portopulmonary hypertension. The role of sildenafil (or anti-proliferative agents) in the earliest presentation of portopulmonary hypertension is unknown. Questioning the effectiveness of anti-platelet aggregation and anti-proliferative approaches in combination with a pulmonary vasodilator therapy would be reasonable hypotheses. Inclusion of portopulmonary hypertension patients in randomised controlled trials with portopulmonary hypertension subgroup analysis would make additional sense. In appropriately selected patients, early medical treatment of the vaso-obstructive pulmonary vascular process, followed by timely liver transplantation to correct the hepatic/portal pathophysiology that facilitates the evolution of pulmonary arterial hypertension, may provide not only control, but even a cure for portopulmonary hypertension. Optimal treatment for portopulmonary hypertension remains an unanswered, but important question.

\section{REFERENCES}

1 Rodriguez-Roisin R, Krowka MJ, Hervé P, Fallon MB. Pulmonary-hepatic vascular disorders. Eur Respir J 2004; 24: 861-880.

2 Murray KF, Carithers RL Jr. AASLD practice guidelines: evaluation of the liver transplant patient. Hepatology 2005; 41: 1407-1432.

3 Humbert M, Sitbon O, Chaouat A, et al. Pulmonary arterial hypertension in France: results from a national registry. Am Rev Respir Crit Care Med 2006; 173: 1023-1030.

4 Reichenberger F, Voswinckel R, Steveling E, et al. Sildenafil treatment for portopulmonary hypertension. Eur Respir J 2006; 28: 563-567.

5 Galie N, Ghofranu HA, Torbicki A, et al. Sildenafil citrate therapy for pulmonary arterial hypertension. $N$ Engl J Med 2005; 353: 2148-2157.

6 Krowka MJ, Frantz RP, McGoon MD, Severson C, Plevak DJ, Wiesner RH. Improvement in pulmonary hemodynamics during intraveneous epoprostenol (prostacyclin): a study of 15 patients with moderate to severe portopulmonary hypertension. Hepatology 1999; 30: 641-648.

7 Hoeper MM, Halank M, Marx C, et al. Bosentan therapy for portopulmonary hypertension. Eur Respir J 2005; 25: 502-508. 